J Comp Physiol A (1985) 156:539-545

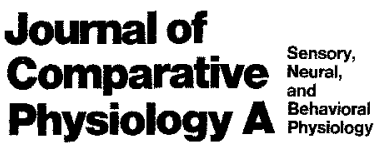

(C) Springer-Verlag 1985

\title{
The effects of feeding on heart activity in the terrestrial slug, Limax maximus: central and peripheral control
}

\author{
Debra S. Grega* and David J. Prior \\ Physiology Group, School of Biological Sciences, University of Kentucky, Lexington, Kentucky 40506, USA
}

Accepted January 2, 1985

\begin{abstract}
Summary. The role of the central nervous system (CNS) in the modulation of heart activity induced by feeding was investigated in the terrestrial slug, Limax maximus. Intact slugs and semi-intact preparations were used to examine the effects of food, non-nutritive bulk, digestive tract distension, and the concentration of hemolymph glucose on the control of heart activity. The heart rate of intact slugs increased following ingestion of food or nonnutritive bulk and in response to injections of glucose. The heart rate of semi-intact preparations increased in response to gradual crop inflation and to perfusion of the heart with a glucose solution for longer than $30 \mathrm{~min}$. The present results indicate that the increase in heart rate observed in intact slugs following a meal is mediated in part by the CNS and in part is a direct response of the heart musculature. The CNS mediates an immediate response to proprioceptive input from stretch of the crop while the heart musculature responds directly to increased hemolymph glucose concentration following ingestion of food.
\end{abstract}

\section{Introduction}

Molluscs have myogenic hearts, the rhythmic contractions of which are the result of the pacemaker activity of specialized cardiac muscle cells (Prosser 1973). Cardiac activity can be modified in response to numerous internal and external stimuli such as temperature, state of hydration and tactile stimulation. This modulation can involve direct effects on the heart musculature or may be mediated

\footnotetext{
* Present address: Neuroscience Laboratory Bldg., 1103 East Huron, University of Michigan, Ann Arbor, MI 48109, USA
}

by the central nervous system (CNS). CNS modulation occurs via central cardioregulatory neurons which synapse on heart muscle fibers and modify the frequency and force of contraction (Krijgsman and Divaris 1955; Irisawa 1978). Peripheral modulation, which does not involve the CNS, can occur when the cardiac muscle cells respond directly to changes in the internal environment (Irisawa 1978; Grega and Prior 1985). The ingestion of food has numerous effects on an animal, including an increase in oxygen consumption and metabolic rate (Prosser 1973).

The effect of food intake on cardiac activity has been investigated in the marine opisthobranch, Aplysia californica, by Dieringer et al. (1978) and the terrestrial pulmonates Deroceras reticulatum (Duval 1983) and Limax maximus in the present study. In intact Aplysia, Dieringer et al. (1978) reported that an increase in heart rate was associated with arousal to food and continued during and following a meal. Only the increase in heart rate that was associated with food arousal seemed to be neuronally mediated. Duval (1983) measured the heart rate of intact Deroceras by direct observation of the cardiac pulsations. He observed a $30 \pm 9 \%$ increase in heart rate accompanying the onset of feeding. He interpreted this as being unrelated to digestion due to its rapid onset and proposed that it was due to the necessity of raising hemocoelic pressure for mouth protrusion. Duval did not, however, investigate the possibility that the nervous system was involved in the initiation of the increase in heart rate. Using Limax, we have investigated the role of the central nervous system in mediating feeding-induced modulation of heart activity. It was possible that alterations in heart activity involved cardioregulation via the CNS. In addition, the heart musculature could respond di- 
rectly to feeding related stimuli. Intact slugs and semi-intact preparations were used to examine the effects of food, non-nutritive bulk, digestive tract distension and the concentration of hemolymph glucose on the control of heart activity.

\section{Materials and methods}

Limax maximus were collected locally and kept at room temperature in vented plastic boxes lined with moist paper towels. They were fed Purina rat chow ad libitum except when fasting is specified. Slugs of 2-5 g were used in these experiments. Heart rates in intact animals were determined by visual observation of heart contractions through the pneumostome (an opening to the mantle cavity). When open, the pneumostome permits visualization of the lung and, with proper backlighting, the rhythmic contractions of the heart. The time necessary for ten heart beats was measured and the mean of three individual measurements was used to express heart rate in beats per minute.

Semi-intact preparations were used consisting of heart, nerve 11 (N11, Grega 1982; visceral nerve, MacKay and Gelperin 1972) and brain. Cutting N11 did not significantly alter the heart rate, suggesting no tonic CNS input for normal heart contraction. For the semi-intact preparation, the heart was left undisturbed atop the kidney due to their close apposition and innervation. Most of the lung also was left attached to the heart and kidney to ease heart perfusion and stabilization of the preparation in the dish. The heart was perfused with a constant pressure gravity feed system by cannulating one of the major anastomosing pulmonary veins in the lung. In some experiments, the anterior gut (crop, esophagus and buccal mass) was kept attached to the heart-N11-brain preparation, care being taken to not disrupt innervation. The gut was cut at the crop-stomach junction and a polyvinylchloride cannula inserted into the crop and secured with a ligature. The cannula was attached to a syringe for inflation and deflation of the crop. Another anterior ligature not was necessary to keep crop contents from refluxing out the buccal mass during inflation.

Mechanical activity of the heart in the semi-intact preparations was monitored by attaching the ventricle to a Grass FT.03C force transducer via a small hook. The transducer was connected to the D.C. preamplifier of a Grass model 79D polygraph. In semi-intact preparations, the heart rate was determined in two ways: either counting the number of heart contractions within a $15 \mathrm{~s}$ interval or for instantaneous rate, by using the intercontraction interval (for comparison with data for Aplysia, Dieringer et al. 1978). Heart rates were used to calculate the mean rate, standard deviation (S.D.) and standard error (SEM in intact animals when the heart rate was the mean of 3 individual observations). When pooling data, variations between individual animals were minimized by expressing changes as percent change from control values. Nonparametric statistics were applied to these data when expressed as percentages. To determine significant differences, the Mann Whitney $U$-test for unpaired data and the Spearman rank coefficient were used to determine significant correlation. When using actual heart rates, a $t$-test was used to determine significant differences.

The normal saline had a final composition, in $\mathrm{mmol} / \mathrm{l}$, of $55.6 \mathrm{Na}^{+} ; \quad 4.2 \mathrm{~K}^{+} ; \quad 7.0 \mathrm{Ca}^{++} ; \quad 4.6 \mathrm{Mg}^{++} ; \quad 80.3 \mathrm{Cl}^{-}$; $0.2 \mathrm{H}_{2} \mathrm{PO}_{4}^{-} ; 5.0 \mathrm{HCO}_{3}^{-}$and 5.0 glucose; and an osmolality of $145 \mathrm{mOsm} / \mathrm{kg} \mathrm{H}_{2} \mathrm{O}$. In glucose-free and fructose salines, the glucose was omitted. The fructose saline contained $1.0 \mathrm{mmol} / \mathrm{l}$ fructose. The filtered carrot infusion was prepared from a mixture of $10 \mathrm{~g}$ of grated carrot and $20 \mathrm{ml}$ of distilled water which were combined for $5 \mathrm{~min}$ prior to filtering.
Table 1. Effect of gut distention on heart rate of intact slugs

\begin{tabular}{lcc}
\hline Meal & $\begin{array}{l}\text { Percentage increase } \\
\text { in weight }\end{array}$ & $\begin{array}{l}\text { Percentage increase } \\
\text { in heart rate }\end{array}$ \\
\hline $\begin{array}{lcc}\text { Food } \\
(n=16)\end{array}$ & $7.2 \pm 7.1$ & $16.7 \pm 11.7$ \\
$\begin{array}{l}\text { Non-nutritive bulk } \\
(n=16)\end{array}$ & $4.5 \pm 2.9 \pm 2.9$ & $8.1 \pm 2.2$ \\
$(1.1 \pm 11.0)$ & $(4.3-12.6)$ \\
\hline
\end{tabular}

The ingestion of food or non-nutritive bulk on heart rate of intact slugs was determined. Data are expressed as the mean \pm SEM with the range (minimum - maximum) values. Meal size expressed as percentage increase from fasted body weight. There are statistically significant correlations between weight increase and increase in heart rate (Spearman rank coefficient, for food $P=0.05$; for non-nutritive bulk $P=0.01$ )

Glucose concentrations were determined for slug hemolymph, filtered carrot infusion and filter paper infusion (distilled water in which Whatman Qualitative Filter paper had soaked). Blood samples $(0.01 \mathrm{ml})$ were collected from a cut superior tentacle and were immediately frozen. When thawed, blood samples were centrifuged in a Beckman 3200 centrifuge for $30 \mathrm{~min}$ at $12,800 \times \mathrm{g}$ before the glucose concentration was measured using the Sigma Enzymatic Colorimetric Glucose Test (Sigma Chemical Co.). The optical density of the solutions was measured at $420 \mathrm{~nm}$ on a Klett-Summerson Photoelectric colorimeter. A $16 \mathrm{mmol} / 1$ glucose solution was used as a glucose standard.

\section{Results}

In the first experiment, the heart rates of fasted slugs (14 d) were compared with their heart rates two hours after feeding. Following a meal there was a $16.7 \pm 11.7 \%$ increase in heart rate (Table 1 ). There was a significant correlation between the increase in heart rate and the increase in body weight due to the meal (significant at $P=0.05$, Spearman rank coefficient). To determine the time course of the increase in heart rate after a meal, the postmeal heart rates of nine slugs were compared with their premeal rate (mean meal duration $=12.1 \pm 2.1 \mathrm{~min}$ (SD), $n=9$; Fig. 1). There was an initial increase which lasted $90 \mathrm{~min}$, after which heart rate began to decline to premeal levels. The increase in heart rate after a meal may be due to several factors: (1) gut distention could result in sensory feedback to the CNS which could, in turn, modulate heart rate, (2) an increase in the levels of nutrients in the blood may have an effect on the CNS, directly on the heart or both, (3) the mechanical work involved in digestion and the increase in metabolic rate associated with a meal could initiate an increase in heart rate due to increased oxygen demand. The effect of stretch of the gut was examined in intact slugs and semi-intact preparations. The heart rates of intact slugs were compared be- 


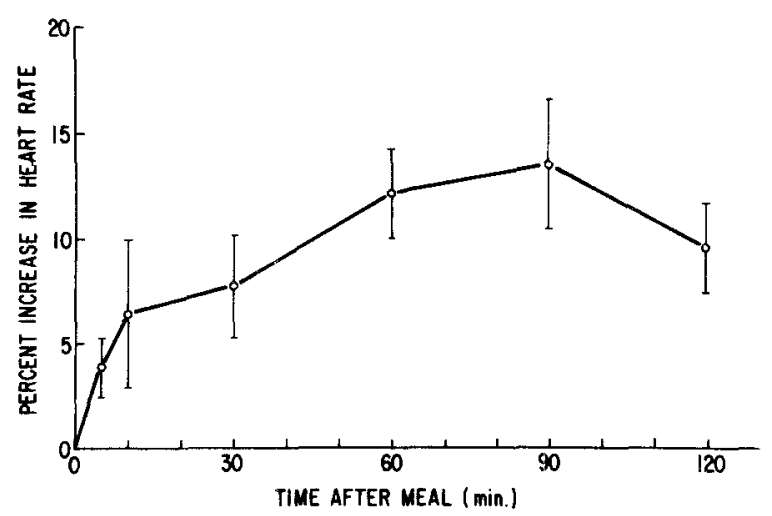

Fig. 1. Effect of food intake on postmeal heart rate. In intact slugs, postmeal heart rate was compared with the fasted (14 days; premeal) heart rate. The values plotted are the mean \pm 1 SEM for 9 slugs, one measurement per animal per time point

fore, and $2 \mathrm{~h}$ after a meal of non-nutritive bulk (filter paper). It had been observed previously that slugs fasted for long periods would eat filter paper (Sokolove et al. 1977). To enhance prompt ingestion of the filter paper upon presentation to the slug, the paper was dampened with filtered carrot infusion. The filter paper did not contain a significant amount of glucose as determined with the glucose assay. The carrot infusion contained a slight amount of glucose $\left(2.7 \times 10^{-4} \mathrm{mmol}\right.$ per dampened filter paper), however, when the amount of glucose ingested was calculated, based upon the mean filter paper meal size $(0.123 \mathrm{~g})$, it represented only about $4 \%$ of the hemolymph glucose level $\left(3.2 \times 10^{-5} \mathrm{mmol}\right.$ glucose from the carrot infusion as compared with $6.9 \times 10^{-4} \mathrm{mmol}$ glucose in the hemolymph of the fasted slug). Intact slugs were fasted for 28 days, kept in plastic containers with no paper towels and only water. After fasting, 16 of 20 animals ate filter paper within $30 \mathrm{~min}$ of presentation. There is a significant correlation between the increase in weight from feeding on nonnutritive bulk and the increase in heart rate (at $P=0.01$, Spearman rank coefficient; Table 1). Thus, filling of the digestive tract, regardless of the nutritional value of the meal results in an increase in heart rate.

To further investigate the effect of crop distention, a semi-intact preparation of the heart, brain and anterior gut (crop, esophagus and buccal mass) was used. Reingold and Gelperin (1980) demonstrated that sensory information from inflation of the crop caused early termination of the feeding motor program in a semi-intact preparation. This proprioceptive feedback to the CNS could modulate cardiac as well as feeding activity.
A
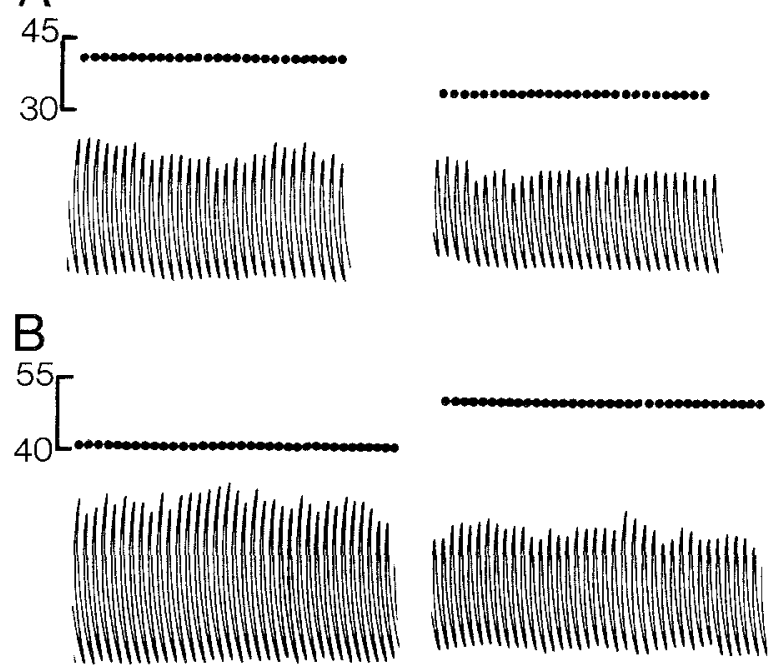

$-1$

Fig. 2 A, B. Effect of crop inflation on heart rate. In an isolated preparation, the crop was inflated with saline $(5 \%$ volume/body weight). In $\mathbf{A}$, inflation time was rapid (10 s) whereas in $\mathbf{B}$, it was slow (16.6 min). In both $\mathbf{A}$ and $\mathbf{B}$, the top graph represents instantaneous heart rate as calculated from intercontraction interval. The second trace depicts the mechanical activity of the heart. The left portion of both $\mathbf{A}$ and $\mathbf{B}$ illustrate the control heart rate with the crop deflated. The right portion depicts heart rate at completion of crop inflation $(10 \mathrm{~s}$ in $\mathbf{A}$; 16.6 min in $\mathbf{B}$ ). Rate changes in both $\mathbf{A}$ and $\mathbf{B}$ are significantly different from control values $(P=0.001 ; t$-test $)$. Calibration bars: vertical $20 \mathrm{mg}$; horizontal $5 \mathrm{~s}$

To test this, the gut was cut at the crop-stomach junction and a volume of glucose-free saline equal to $5 \%$ of the body weight $(5 \% \mathrm{vol} / \mathrm{IBW})$ was injected to distend the crop. The mean increase in weight due to a meal was $9.1 \pm 0.3 \%$ of IBW. This was determined by weighing the anterior gut of fasted slugs $(10 \mathrm{~d})$ before or after completion of a meal. The anterior gut was tied off at the stomach-crop junction or the entire digestive tract was removed intact and weighed. The crop then was opened, the contents removed and anterior gut reweighed. The weight of the crop contents in fasted slugs was $5.6 \pm 0.9 \%$ fasted body weight $(n=3)$ and $16.5 \pm 0.2 \%$ of the body weight $(n=4)$ in slugs immediately after feeding. Rapid ( $10 \mathrm{~s} \mathrm{du}-$ ration) crop inflation with saline resulted in a decrease in heart rate (Fig. $2 \mathrm{~A}$; Table $2 \mathrm{~A}$ ). In that most abrupt stimuli result in cardioinhibition (Grega 1982), the decrease in heart rate was not unexpected. To more accurately mimic the crop distention that occurs during a meal, the crop was gradually inflated (Fig. 2B; Table 2A). Slow crop inflation over periods of 15 to $20 \mathrm{~min}$ resulted in a $6.0 \pm 2.7 \%(\mathrm{SD} ; n=8$; at $P=0.02$, Mann Whit- 
Table 2. Effect of crop inflation on heart rate

\begin{tabular}{llll}
\hline $\begin{array}{l}\text { Type of } \\
\text { inflation }\end{array}$ & Type of saline & $\begin{array}{l}\text { Percentage change } \\
\text { in heart rate }\end{array}$ & $n$ \\
\hline A. Rapid & Glucose-free & $6.5 \pm 2.4$ decrease & 4 \\
Slow & Glucose-free & $6.0 \pm 2.7$ increase & 8 \\
B. Slow & Glucose-free & $5.8 \pm 1.9$ increase & 5 \\
Slow & 1 mmol/l glucose & $6.1 \pm 1.3$ increase & 5 \\
\hline
\end{tabular}

The percentage change in heart rate was determined by comparing the change in heart rate with crop inflation with that when the inflation was done with the crop untied or punctured and therefore not inflatable. All heart rate increases were statistically significant (Mann Whitney, $P=0.2$ )

ney) increase in heart rate. After a 10 min interval, when N11 was cut and the crop reinflated, there was no alteration in heart rate, an observation that strongly suggests involvement of the CNS.

The $6.0 \%$ increase in heart rate that resulted from crop distention was not sufficient to entirely account for the increase in heart rate observed following a meal. It is possible that the increased level of nutrients from the ingested food had an effect on heart rate as well. To test this alternative, a series of experiments were done using intact and semiintact animals: (1) hemolymph glucose levels were measured in fasted and fed slugs. (2) Heart rate was measured in fasted slugs injected with glucose. (3) In an isolated preparation, the heart and brain were sequentially exposed to glucose while the heart rate was monitored. (4) The crop was distended with $1 \mathrm{mmol} / 1$ glucose saline, thus combining crop stretch with increased glucose concentration. (5) The heart was perfused with glucose analogs to determine their site of action on the heart.

To determine if a meal can significantly alter hemolymph glucose levels, the glucose concentration of the blood was measured in fasted $(7 \mathrm{~d} ; n=$ 9) and fed $(n=6)$ slugs $1 \mathrm{~h}$ after completion of a meal of Purina rat chow. The mean meal duration for the fed slugs was $9.4 \pm 3.5 \mathrm{~min}(\mathrm{SD})$. There was a five fold increase in hemolymph glucose concentration from $0.26 \pm 0.1$ to $1.32 \pm 0.57 \mathrm{mmol} / \mathrm{l}$ (at $P=0.01$, Mann Whitney) $1 \mathrm{~h}$ after the meal.

The effect of glucose on the heart rate of intact animals, was investigated by the injection of fasted slugs with a volume of $0.1 \mathrm{~mol} / 1$ glucose saline calculated to raise the hemolymph glucose concentration to $4 \mathrm{mmol} / 1$. The heart rate was monitored by direct visual observation through the pneumostome. Control animals were injected with a comparable volume of glucose-free saline. The effect of increased glucose concentration was biphasic, initially causing a decrease in heart rate, but after

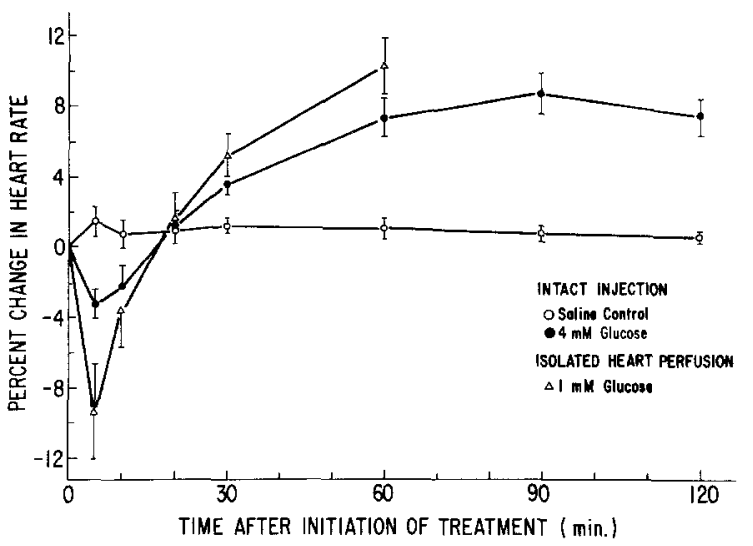

Fig. 3. Alterations in heart rate in intact slugs and the semiintact preparation in response to glucose. Changes in heart rate, expressed as percent change from control rate, are plotted versus time after initiation of glucose treatment. Circles represent the percent change in heart rate of intact, fasted slugs which were injected with $4 \mathrm{mmol} / 1$ glucose saline $(\bullet)$ or glucose free saline (control o). Heart rate was determined by observation through the pneumostome. The open triangle $(\Delta)$ represents the heart rates of isolated hearts initially perfused with glucosefree saline for at least $30 \mathrm{~min}$ prior to perfusion with $1 \mathrm{mmol} / \mathrm{l}$ glucose saline. Each point plotted is the mean of 10 individuals (intact animals or heart preparations, one measurement per slug per time point \pm 1 SEM). In both the intact and isolated preparations, glucose caused an initial decrease in heart rate followed by an increase in rate

$20 \mathrm{~min}$ switching to an increase in heart rate (Fig. 3). The saline control injections resulted in a slight $(1.9 \pm 0.7 \%)$ increase in heart rate which persisted throughout the experiment.

To ascertain whether the glucose effect observed in the whole animal was mediated by the CNS or the heart itself, an isolated heart-brain preparation was used in which the brain and heart were placed in separate chambers, connected by N11. Either the heart or the brain were exposed to $1.0 \mathrm{mmol} / 1$ glucose saline after being bathed in glucose-free saline. The application of glucose to the heart resulted in an immediate decrease in heart rate with an increase in contraction amplitude (Fig. 3; Table 3). With long-term glucose perfusion of the heart, the biphasic response observed in the whole animal, was seen in the isolated heart (Fig. 3).

To address whether there are glucose-sensitive afferent neurons in the heart whose input to the CNS affects central cardioregulatory neurons, the heart was perfused with glucose-free saline and then perfused with $1.0 \mathrm{mmol} / 1$ glucose saline for $30 \mathrm{~min}$. After washing for $1 \mathrm{~h}$ with glucose-free saline, N11 was cut and the glucose perfusion repeated. There was no difference in the alteration of heart rate in response to glucose before or after cutting N11 (Mann Whitney; $P=0.05 ; n=6$ ). 
Table 3. Perfusion of the isolated heart

\begin{tabular}{lll}
\hline Min & \multicolumn{2}{l}{$\begin{array}{l}\text { Percentage change in heart rate } \pm \text { SEM } \\
\text { Saline }\end{array}$} \\
\cline { 2 - 3 } & $\begin{array}{l}1 \mathrm{mmol} / 1 \text { glucose } \\
(n=10)\end{array}$ & $\begin{array}{l}1 \mathrm{mmol} / 1 \text { fructose } \\
(n=6)\end{array}$ \\
\hline 2 & $14.4 \pm 1.5 \mathrm{Decr}^{\mathrm{a}}$ & $5.0 \pm 2.7 \mathrm{Decr}^{\mathrm{a}}$ \\
5 & $9.5 \pm 2.6 \mathrm{Decr}^{\mathrm{a}}$ & $11.2 \pm 3.9 \mathrm{Decr}^{\mathrm{a}}$ \\
10 & $3.7 \pm 2.1 \mathrm{Decr}^{\mathrm{a}}$ & $11.6 \pm 1.8 \mathrm{Decr}^{\mathrm{a}}$ \\
20 & $1.8 \pm 1.6 \mathrm{Incr}^{\mathrm{a}}$ & $7.9 \pm 0.5 \mathrm{Decr}^{\mathrm{a}}$ \\
30 & $5.5 \pm 1.2 \mathrm{Incr}^{\mathrm{a}}$ & $4.5 \pm 0.6 \mathrm{Decr}^{\mathrm{a}}$ \\
60 & $10.4 \pm 1.7 \mathrm{Incr}^{\mathrm{a}}$ & $1.2 \pm 1.2 \mathrm{Decr}^{2}$ \\
\hline
\end{tabular}

Decr Decrease; Incr Increase

a Indicates that the rate is statistically different than that in control glucose-free saline ( $t$-test, $P=0.05$ )

When the brain was exposed to $1.0 \mathrm{mmol} / 1$ glucose saline while the heart was perfused with glucosefree, the changes in heart rate were not different from the controls in which glucose-free saline was used as the brain perfusion solution (Mann Whitney; $P=0.05 ; n=6$ ).

The isolated heart, brain and crop preparation was used to determine if the combination of crop distension with glucose saline resulted in greater heart rate changes than those seen with crop stretch in glucose-free saline. The crop was gradually distended with glucose-free saline $(5 \% \mathrm{vol} /$ IBW) and then deflated by withdrawing the saline. After $5 \mathrm{~min}$, the crop was gradually reinflated $(5 \%$ vol/IBW) with $1.0 \mathrm{mmol} / \mathrm{l}$ glucose saline. Both $1.0 \mathrm{mmol} / 1$ glucose and glucose-free saline resulted in a $6 \%$ increase in heart rate with crop distension (Table 2B; the treatments were not significantly different at $P=0.05$, Mann Whitney, $n=5$ ).

Finally, to ascertain the site of action of glucose in the heart, the isolated heart was perfused with a variety of substances including glucose analogs. To determine if an active transport system for glucose was involved, the heart was perfused with either a fructose or ouabain saline. Although fructose can be metabolized, it is not transported by the glucose active transport system. The short-term (2 min) effects of fructose perfusion caused a $5.0 \pm 2.7 \%$ (SEM) decrease in heart rate compared to the $14.4 \pm 1.5 \%$ (SEM) decrease observed with glucose (Table 3). The time course of the response was also different, with a decrease in heart rate which was reversed within the hour (Table 3 ). Ouabain, which inhibits $\mathrm{Na}^{+} / \mathrm{K}^{+}$dependent ATPase, blocks active glucose transport in all known carrier systems (Schultz and Curran 1970). Perfusing the heart with $1.0 \mathrm{mmol} / \mathrm{l}$ ouabain in glucose-free saline alone caused an increase in heart rate (Fig. 4).

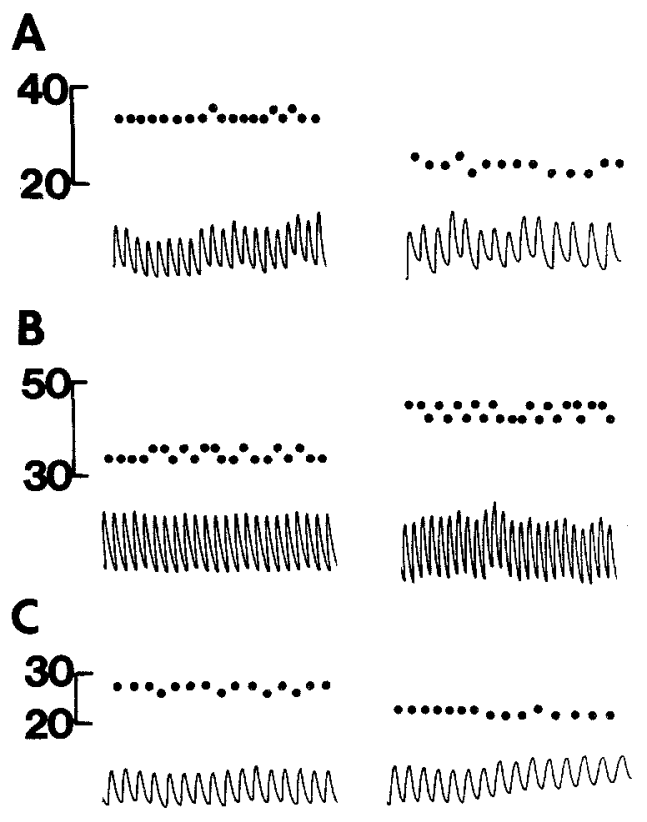

Fig. 4A-C. Effect of ouabain on cardiac response to glucose. The isolated heart was perfused with $1.0 \mathrm{mmol} / \mathrm{lglucose}$ saline in $\mathbf{A}, 1.0 \mathrm{mmol} / \mathrm{l}$ ouabain in glucose-free saline in $\mathbf{B}$ and in C, a saline with $1.0 \mathrm{mmol} / 1$ each of ouabain and glucose. The left portion of each section represents the control heart activity $2 \mathrm{~min}$ before the test perfusion was initiated. The heart was washed with glucose-free saline for 45 to $60 \mathrm{~min}$ between each of $\mathbf{A}, \mathbf{B}$ and $\mathbf{C}$. In $\mathbf{A}, \mathbf{B}$ and $\mathbf{C}$ the top graph represents the instantaneous heart rate as calculated from the intercontraction interval. Second trace depicts the mechanical activity of the heart. Rate changes observed in $\mathbf{A}, \mathbf{B}$ and $\mathbf{C}$ are significantly different than control values (at $P=0.01 ; t$-test). Calibration bars: vertical $20 \mathrm{mg}$; horizontal $5 \mathrm{~s}$

However, $1.0 \mathrm{mmol} / 1$ ouabain plus $1.0 \mathrm{mmol} / 1 \mathrm{glu}-$ cose saline resulted in decreased heart rate and increased contraction amplitude just as glucose alone did (Fig. 4). Thus it appears that active transport of glucose is not necessary for the glucose effect on the heart. The last of the glucose analogs examined was 3-O-Methyl glucose (3OMG) which substitutes for glucose but is not phosphorylated and therefore, is not metabolized (Csáky and Wilson 1956). Perfusion of the heart with $1.0 \mathrm{mmol} / 1$ $3 \mathrm{OMG}$ saline resulted in no change in heart rate or magnitude of contraction compared with that observed in glucose-free saline $(n=6 ; t$-test; $P=$ 0.05 ). Thus, the glucose effect on the heart appears to require a metabolizable sugar and does not require active or facilitated transport of that sugar.

\section{Discussion}

In vertebrates (Brobeck 1979) and many invertebrates (Prosser 1973) there is an increase in heart 
rate following a meal. An exception appears to be the bivalve Mytilus, which demonstrates no change in heart rate following feeding, although oxygen consumption and ventilation do increase (Thompson and Bayne 1972). The postmeal increase in heart rate in Limax appears to be mediated in part by the CNS and in part by a direct response of the heart musculature itself (Fig. 1, Table 1). As seen in Fig. 2, the CNS can mediate an increase in heart rate due to proprioceptive input from stretch of the crop (also Table 2). This distention, regardless of the nutritional value of the meal resulted in an increase in heart rate. The effect of proprioceptive feedback from the gut on termination of feeding has been documented in insects (Gelperin 1967, 1971) and gastropods (Aplysia: Susswein and Kupfermann 1975a, b; Limax: Reingold and Gelperin 1980). Gut distention from a meal activates stretch receptors in the crop which in turn inhibit feeding activity. This negative feedback is mediated by the CNS and, in Limax, contributes to the termination of feeding (Reingold and Gelperin 1980) as well as to increasing the frequency of heart contraction (Fig. 2; Table 2).

The second component of the feeding-induced change in heart rate is the increase in hemolymph glucose. Increased glucose concentration caused increased heart rate in intact slugs and in isolated, perfused hearts (Fig. 3). The time course of this response was the same in isolated hearts as in intact animals. Interestingly, the isolated heart showed a more robust response than the intact animal, especially with regard to the initial decrease in heart rate. This may reflect the absence of additional inputs in the intact animal which have been removed in the isolated preparation. In other studies where preparations with the heart or brain were sequentially exposed to hyperosmotic saline, perfusion of the heart resulted in a decrease in heart rate. This contrasts with the increase in heart rate observed when the brain is exposed to hyperosmotic saline (Grega 1982; Grega and Prior 1985). Thus in the intact animal, the peripheral cardiac response of decreasing heart rate may be modulated by CNS mediated excitation.

Certain components which contribute to the postmeal increase in heart rate in Limax have been identified. Their time courses can account for the majority of the whole animal response. Filling the digestive tract triggers proprioceptive inputs which, via the CNS, provide both immediate (crop inflation, within $16 \mathrm{~min}$ ) and long term (bulk ingestion, $2 \mathrm{~h}$ ) increases in heart rate. The effect of crop input can be considered to be prolonged for the duration of crop distension. Reingold and Gel- perin (1980) reported that crop input to the feeding program appears to be tonic; as long as the crop is inflated, the feeding program is altered. Food can remain in the crop for up to $24 \mathrm{~h}$ (Agriolimax reticulatus, Walker 1969). This tonic excitatory input could mask the initial slight decrease in heart rate in response to increased hemolymph glucose. From the slight depression in the heart rate increase following a meal (Fig. 1), the glucose effect (Fig. 3) is probably initiated $10-30 \mathrm{~min}$ after a meal. Together, the proprioceptive and glucose effects appear to be responsible for the majority of the response observed in the intact animal.

In each of the gastropods studied thus far ( $A p$ lysia, Deroceras and Limax) feeding induces an increase in heart rate. Dieringer et al. (1978) reported that in Aplysia, the increase in postmeal heart rate was not altered by removal of CNS cardiac input, thus implying that it was entirely a direct response of the heart to the change in nutritional state. Duval (1983) suggested that in Deroceras, the increase in rate was not due to digestion, but rather represented the heart's contribution to increasing hemocoelic pressure for proper mouth or odontophore protrusion during feeding. This response may include some CNS involvement although Duval did not pursue that line of investigation. In Limax, unlike Aplysia, the postmeal increase in heart rate is mediated, in part, by the CNS. The cardiac response in Limax results from both an immediate, CNS mediated increase in heart rate and a second, long-term response of the heart musculature to increased hemolymph glucose. Duval's (1983) observations of Deroceras were made only during feeding sessions. A long-term heart rate increase comparable to that observed in Limax may occur in Deroceras. Thus, the cardiac responses of Limax, and perhaps Deroceras as well, demonstrate a basic organizational difference in the control of cardiac function in pulmonate and opisthobranch gastropods.

Acknowledgements. This work was supported by the Whitehall Foundation and an N.I.H. - R.C.D.A. to D.J.P and a Research Fellowship from the University of Kentucky to D.S.G. This is contribution \#228 from the Tallahassee, Sopchoppy, and Gulf Coast Marine Biological Association.

\section{References}

Brobeck JR (1979) Best and Taylor's Physiological basis of medical practice. Tenth ed, Section 3. The Williams and Wilken, Baltimore, pp 235

Csáky TZ, Wilson JE (1956) The fate of $3-\mathrm{C}^{14} \mathrm{CH}_{3}$-glucose in the rat. Biochem Biophys Acta 22:185-186

Dieringer N, Koester J, Weiss KR (1978) Adaptive changes in heart rate of Aplysia californica. J Comp Physiol $123: 11-21$ 
Duval A (1983) Heartbeat and blood pressure in terrestrial slugs. Can J Zool 61:987-992

Gelperin A (1967) Stretch receptors in the foregut of the blowfly. Science 157:208-210

Gelperin A (1971) Abdominal sensory neurons providing negative feedback to the feeding behavior of the blowfly. Z Vergl Physiol 72:17-31

Grega DS (1982) Central and peripheral cardiac regulation in the pulmonate mollusc, Limax maximus. PhD Dissertation, University of Kentucky

Grega DS, Prior DJ (1985) Alterations in cardiac activity in response to dehydration in the terrestrial slug, Limax maximus. J Exp Zool (in press)

Irisawa H (1978) Comparative physiology of the cardiac pacemaker mechanism. Physiol Rev 58:461-498

Krijgsman BJ, Divaris GA (1955) Contractile and pacemaker mechanisms of the heart of molluscs. Biol Rev 30:139

MacKay AR, Gelperin A (1972) Pharmacology and reflex responsiveness of the heart of the giant garden slug Limax maximus. Comp Biochem Physiol 43:877-896
Prosser CL (1973) Comparative animal physiology. Saunders, Philadelphia

Reingold SC, Gelperin A (1980) Feeding motor programme in Limax. II. Modulation by sensory inputs in intact animals and isolated central nervous systems. J Exp Biol 85:1-19

Schultz SG, Curran PF (1970) Coupled transport of sodium and organic solutes. Physiol Rev 50:637-718

Sokolove PG, Beiswanger CM, Prior DJ, Gelperin A (1977) A circadian rhythm in the locomotor behaviour of the giant garden slug, Limax maximus. J Exp Biol 66:47-64

Susswein AJ, Kupfermann I (1975a) Bulk as a stimulus for satiation in Aplysia. Behav Biol 13:203-209

Susswein AJ, Kupfermann I (1975b) Localization of bulk stimuli underlying satiation in Aplysia. J Comp Physiol $101: 309-328$

Thompson RJ, Bayne BL (1972) Active metabolism associated with feeding in the mussel Mytilus edulis. J Exp Mar Biol Ecol 9:111-124

Walker G (1969) Studies of digestion of the slug Agriolimax reticulatus (Miller). (Mollusca, Pulmonata, Limacidae). PhD Dissertation, University of Wales 